\title{
Conditional inspection and maintenance of âsystem with two interacting components
}

\author{
Berrade, M.D. ${ }^{1}$, Scarf, P.A. ${ }^{2}$ and Cavalcante C.A.V ${ }^{3}$ \\ ${ }^{1}$ Departamento de Métodos Estadísticos, Universidad de Zaragoza, Spain. \\ 2 Salford Business School, University of Salford, Manchester M5 4WT, UK. \\ ${ }^{3}$ Universidade Federal de Pernambuco, Department of Engineering Management, Brazil \\ Abstract
In this paper we consider the inspection and maintenance of a two-component \\ system with stochastic dependence. A failure of component 1 may induce the defec- \\ tive state in component 2 which in turn leads to its failure. A failure of component \\ 1 and a defect in component 2 are detected by inspection. Our model considers a \\ conditional inspection policy: when component 1 is found to have failed, inspection \\ of component 2 is triggered. This opportunistic inspection policy is a natural one \\ to use given this stochastic dependence between the components. The long-run cost \\ per unit time (cost-rate) of the conditional inspection policy is determined gener- \\ ally. A real system that cuts rebar mesh motivates the model development. The \\ numerical examples reveal that when the ratio of the cost of corrective system re- \\ placement, that is on failure, to the cost of preventive system replacement is large \\ there exists a finite optimum policy in most cases. Moreover, for the studied system \\ wherein inspections of component 2 are expensive relative to those of component 1 , \\ having a reliable indicator of the defective state in component 2 is a good strategy \\ to avoid costly failures of component 2, particularly when its time to failure is short.
}

Keywords: Maintenance; Inspection; Delay-time; multi-component system; Induced failure; Stochastic dependence; Optimum policy 


\section{Introduction}

Maintenance cost-effectiveness has a direct effect on the competitiveness of organizations [14] and a large part of company budgets is devoted to maintenance and failure prevention [11]. Therefore, the development of models of maintenance decision making is an important area, and the number of published studies is large and growing quickly (Wang [33], Panagiotidou [22], Liu et al. [16]). Nonetheless, Heng et al [14] point to the modeling of component interactions as an area for development. Interactions arise in systems consisting of several components whereby the operation and maintenance of one component influences the operation and maintenance requirements of others. Models that suppose components are independent are, to a greater or lesser extent, deficient; in practice, maintenance that is carried out on failed or degraded components ignoring the effects of such failures or degradation on other components will fail to accomplish its intended effect. Sun et al. [31] argue that interactions commonly occur in engineering assets, especially in mechanical systems. In our paper, we address this need for modeling development by proposing a new model of maintenance in which: if one component is failed then another, because it may be affected by this failure, is inspected; and further the extent of the maintenance to the system depends on the state of the second component. In this way, the model and the policy we propose capture the behaviour of interacting systems and therefore have important managerial implications.

Component interaction or dependence may be of three types: economic, structural, and stochastic (see e.g. Cho and Parlar [9], Dekker et al. [12], Wang [33], Nicholai and Dekker [20]). Economic dependence exists if the cost of combining maintenance differs from the sum of the costs of performing maintenance separately on components, through for example, shared set-ups. Structural dependence exists if components structurally form a sub-system, so that performing a maintenance action on one component necessitates performing maintenance actions on other components, whatever the maintenance actions performed on each component (e.g. replacement, inspection, disassembly and assembly), which themselves need not be the same. Stochastic dependence exists if the state of one component influences the state of other components. For example, Nakagawa [19] models two types of stochastic dependence for a two-component system. In the first, the failure of component one induces a failure of component two with probability $p$. In the second, the failure of component one causes damage to component two. In Satow and Osaki [24], each failure of component one causes a random amount of damage to component two, leading to its failure when the total damage exceeds a specified level. In Lai and Chen [15], failure of component one increases the failure rate of component two. A review of condition-based maintenance for systems with multiple dependent components can be found in Olde Keizer et al [21]. 
The present work is the development of ideas on maintenance policies for systems of interacting components in, for example, Scarf and Deara ([25], [26]). These papers investigate age and block replacement type policies rather than inspection policies and are themselves developments of early ideas on failure interaction discussed by Murthy and Nguyen [18]. Collectively this work deals with failure dependence whereby failure of one component induces a failure in the other with a particular probability. Other work such as Barros et al. [6] or Sheu et al. [29] is somewhat different. In the former, a two-component system with a parallel structure is continuously but imperfectly monitored. The latter presents an age-replacement policy for a two-unit system with failure rate interactions.

Turning to the issue of inspection of multi-component systems, there have been recent advances across the different types of models that are concerned/with determining system state ([35], [28]). For systems in continuous operation, a failure of operational function is detected as soon as it occurs. Protection or cold stand-by systems that operate only on demand must be inspected because a failure is hidden until the point of demand. Inspection distinguishes the good and defective states in systems wherein the intermediate (defective) state is indicative of imminent failure or degraded performance; this notion is the basis of the delay-time models (see Christer [10], Wang [36]). The delay-time model has been used to model the inspection of two-component systems (e.g. Lu et al. [17] ). The delay time concept in the context of multi-component systems is reviewed by Wang et al. [35]. Soft failures are another modelling notion that arise in the context of multicomponent systems and that facilitate the consideration of inspection (e.g. Taghipour et al. [32]). A number of authors have considered inspection policies for two-component systems with failure interaction. Zequeira and Bérenguer [37] study a two-component standby system where the failure of one component can modify the failure probability of the surviving component. Badía et al [3] consider unrevealed failures for a stand-by multicomponent system assuming both economic interaction and dependence between times to failure of components. In, Golmakani and Moakedi [13] failures are stochastically dependent, however, the specification of the interaction is different, with a failure of one component increasing the failure rate of the other. In Wang et al. [35] there exists economic dependence, where failure of one component is an opportunity to inspect others within the same subsystem, but not failure dependence. Berrade et al. [7] is concerned with the inspection and maintenance of a two-component system, assuming a bivariate distribution of times in the good state. However, the maintenance policy they consider is such that the system is effectively a one-component system and they do not make any progress with a two-component system generalization.

In our paper we consider both economic dependence and stochastic dependence in the context of inspection maintenance of a two-component system. We not only suppose that a failure of one component is an opportunity to inspect another but also that failure of one 
may induce a defect in another. Interaction between failures and defective states in this way is a realistic assumption and actual examples can be found in engineering systems. For example, failures of secondary cooling systems can induce defects in primary systems that can in turn lead to failure. Electricity distribution systems (Golmakani and Moakedi, [13]), clutches in semi-automatic gearboxes (Scarf and Deara, [26]), manufacturing systems (Lai and Chen, [15]) provide other examples. Our model is motivated by the maintenance of an electro-mechanical clutch that is part of a guillotine whose function is to cut rebar mesh (steel lattice used to reinforce concrete). This context is described in the next section. In section 3 we define our model of failure and the inspection and maintenance policy. In section 4, we formulate the cost-rate (long run cost per unit of time) for the policy. We then present some results that relate to our case study context. In the final section, we discuss the implications of our work for researchers in the field and for maintainers working in practice.

\section{Case study description}

To illustrate the use of the model in practice, we consider the billet production of rebarmesh, a lattice of welded steel bar used to reinforce concrete. The steel mesh is produced by feeding longitudinal and transverse wires, welded one over the other. The entire process takes place continuously, and after reaching a required size, the mesh must be cut using a guillotine. The movement of a large wheel provides to the guillotine enough force to cut the steel mesh. For this to occur at the appropriate instant, the wheel is coupled and uncoupled from the guillotine by an electro-mechanical clutch. The cutting mechanism must provide power to cut 30 strands of wire of diameter ranging from $3.4 \mathrm{~mm}$ to 6.0 $\mathrm{mm}$. In a broad sense, the electromechanical clutch consists of two parts or components. A static part (component 1) is connected to the guillotine. A mobile part (component 2) is fixed to the driving wheel. Contact between the two parts operates the guillotine. The static part comprises a disc that contains a circular set of tablets, which themselves enable contact with the mobile clutch disc on actuation of the clutch. When the clutch assembly static disc fails, mostly by breaking of the tablets, this failure may damage the mobile disc, since the contact pins can score or crack the mobile clutch disc. Failure of the static disc is only verified by inspection since tablet failure is not considered critical. Furthermore, intermediate states (defects) of the static disc are not identifiable. On the other hand the clutch assembly mobile disc is much more expensive than the static disc, and when the mobile disc fails its repair takes priority over production. Furthermore, it is possible to identify intermediate states or defects in the mobile disc (generally scratches and cracks on the mobile clutch disc) by means of liquid-penetrant inspections using magnetic particles. 
The inspection of the static disc (inspection of the tablets) is straightforward, but their replacement requires time and resources. During the procedure for replacing the tablets, either due to failure or preventively, the engineers are inclined, thus conditionally and opportunistically, to inspect the mobile disc to take advantage of the fact that the system is already stopped. Therefore, in essence, this two-component system is subject to a conditional inspection policy such that:

- when component 1 fails, this failure may damage component 2, and failure of component 1 can only be observed through inspection;

- component 2 is more expensive and more robust than component 1 , and when component 2 fails its repair takes priority over production;

- at inspection of component 1 , if it is found to be failed it may be worthwhile to inspect component 2 and furthermore to replace component 2 if it is defective.

In this sense, failure of component 1 may be considered as a soft failure, as its failure does not directly lead to production stoppage. However when component 1 is failed the production experiences some quality reduction which is not observed by operators and therefore a penalty cost is derived when component 1 fails. In addition, its failure can indirectly lead to production stoppage and so this type of failure is somewhat different to the soft failures discussed by others (e.g. Taghipour et al. [32]). The 2-component view is a simplification of the system for the purpose of modelling and for the quantitative decision support that follows from such modelling. The static disc tablets may themselves be considered as individual components with failure defined in a k-out-of-n manner. However, the service engineers are inclined to replace the static disc as an entire unit due to its relatively low unit-cost. In this way, appropriate service interventions define the scale of resolution of the system in the manner discussed in Scarf et al [27]. Thus, implicit in our discussion are also the ideas of Ascher and Feingold [1] that: components are elements of a maintained system whose lifetime can be appropriately modelled with a probability density function and for which it is reasonable to investigate a bespoke maintenance task; a socket is the interface of a component with the maintained system; and the system is the totality of sockets and components and their interrelationships.

\section{Model specification}

The model contains a number of random variables, decision variables and parameters. The following list presents them to simplify the reading. 


\section{Notation:}

- Random variables:

$X$ : time to failure of component 1 with pdf $f(x)$.

$Y$ : sojourn in the defective state until failure (delay-time) of component 2 and pdf $g(y)$.

$\tau$ : length of a renewal cycle.

$H$ : number of failures of component 1 not inducing a defective state in component 2 that occur in a cycle. For a finite inspection interval, $H$ is also the number of replacements of component 1 in a renewal cycle not including the replacement of component 1 when the system is replaced.

$K_{1}$ : number of inspections of component 1 in a renewal cycle.

$K_{2}$ : number of inspections of component 2 in a renewal cycle, including the inspection at renewal.

$A$ : indicator function of a renewal cycle ending on inspection.

$Z$ : the excess time, the elapsed time from the last inspection of component 1 where component 2 was not defectiye to failure of component 2 .

$D$ : penalty time in a renewal cycle.

$C(\tau)$ : cost of a renewal cycle.

- Decision variable:

$T$ : inspection interval.

- Parameters:

$c_{1}$ is the cost of an inspection of component 1 ;

$c_{2}$ is the cost of an inspection of component 2 (incurred only if component 1 is failed);

$c_{p}$ is the cost of a replacement of component 1 when at inspection component 2 is good;

$c_{I}$ is the cost of a system replacement when at inspection component 2 is defective $\left(c_{p}<c_{I}\right)$;

$c_{F}$ is the cost of a system replacement when component 2 fails $\left(c_{I}<<c_{F}\right)$; 
$c_{d}$ is the penalty cost per unit time incurred while component 1 is failed until it is replaced by a new one or the whole system is replaced whichever occurs first.

Consider a two-component system operating in continuous time. The system is new (both components are good) at time $t=0$. At time $X=t$, component 1 fails. With probability $p$, this failure immediately induces a defect in component 2. With probability $1-p$, component 2 is not affected by failure of component 1 . This is the stochastic failure dependence of the title of this paper. Thus, component 2 remains in the good state until a transition to the defective state is induced by failure of component 1 . Component 2 remains in the defective state for time $Y$, a random variable with density $g(y)$, whereupon it fails. We call this sojourn in the defective state $Y$ the delay time (Christer [10]).

Failures of component 1 are unrevealed. Failures of component 2 are detected as soon as they occur. The failed state of component 1 and defective state of component 2 can only be detected by inspection. We suppose that inspection of component 1 is simple and cheap whereas inspection of component 2 requires more effort and incurs a higher cost. Thus there is a hierarchy (a minor and a major component) in both the cost structure and the failure process of this system. There can be many replacements of component 1 before component 2 is renewed.

We consider the following "conditional" inspection policy: inspect component 1 at times $k T, k=1,2, \ldots$ from new; if component 1 is failed then inspect component 2; if component 1 is good then do not inspect component 2. The possible outcomes at an inspection (state of component 1, state of component 2) are (good, good), (failed, good) and (failed, defective). Then, if the system state at inspection is (good, good), continue operation until next inspection or failure of component 2 whichever occurs sooner; if the system state is (failed, good), then replace component 1 and continue operation until next inspection or failure of component 2 whichever occurs sooner; if the system state is (failed, defective), replace the system. The system is also replaced on failure of component 2. Inspections and replacements are instantaneous. Figure 1 describes a sample path of the maintenance process that ends with the replacement system when component 2 is found to be defective (Figure 1a) or when component 2 fails before the defective state that precedes the failure is discovered on inspection (Figure1b).

We consider that successive component 1 failure times, $X_{1}, X_{2}, \ldots$ are independent and identically distributed (iid) with density function $f(x)$. We assume that system replacements are renewals. Also we assume that $X_{j}$, and $Y$ are independent for all $j$.

We assume that a penalty cost is incurred while component 1 is in the failed state; this is modelled as a cost-rate (as in Golmakani and Moakedi [13] ) so that if component 1 is failed for time $\delta$ then the additional cost incurred is $c_{d} \times \delta$, regardless of whether component 2 is good or defective. Our purpose is to determine $T$, the inspection interval, 
such that the long-run cost per unit of time (cost-rate) is minimized. Therefore in what follows we find expressions for the mean length and mean cost of a renewal cycle ([23]). We do this for a number of scenarios. In the base scenario, the cost structure is as above.

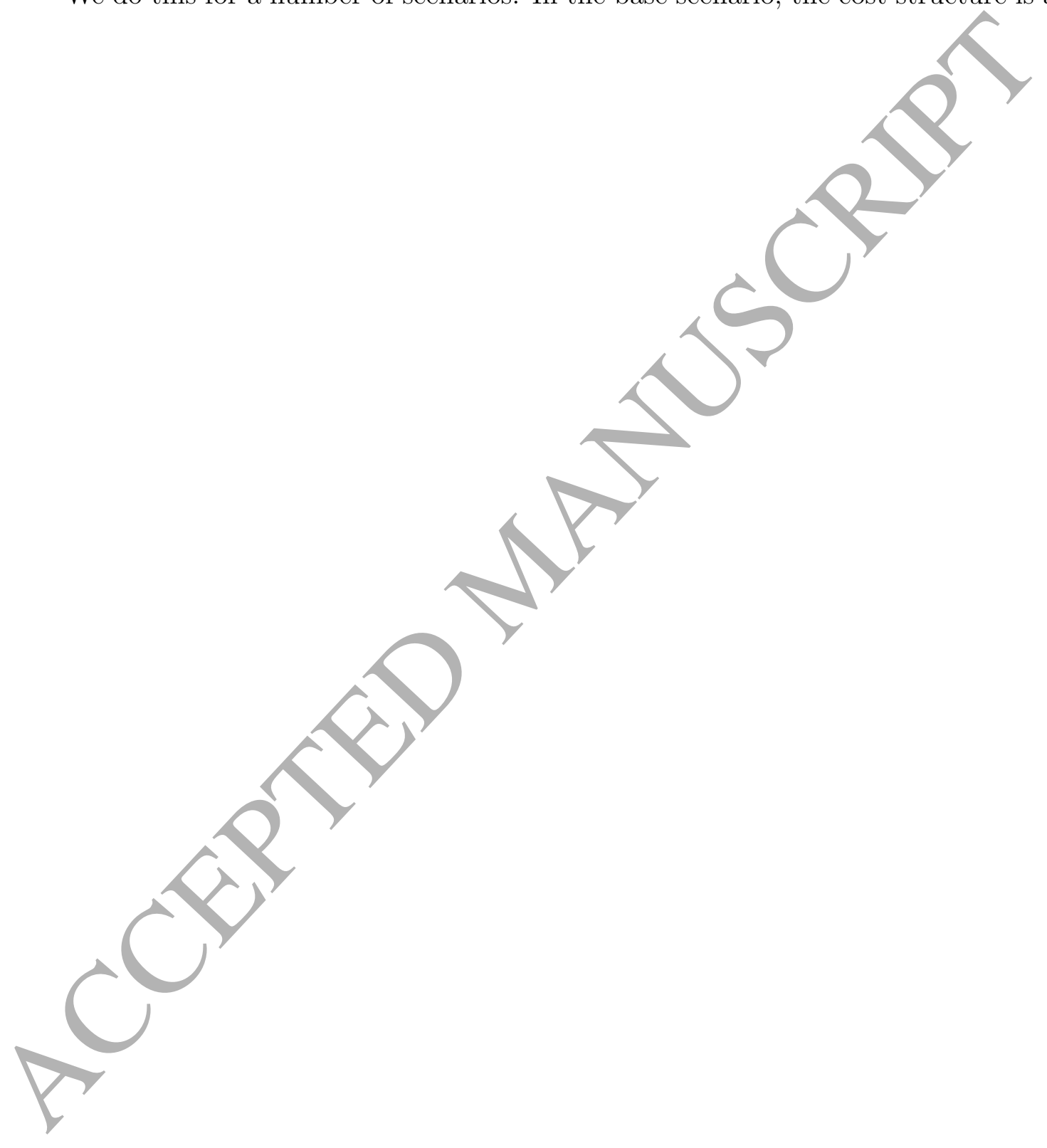




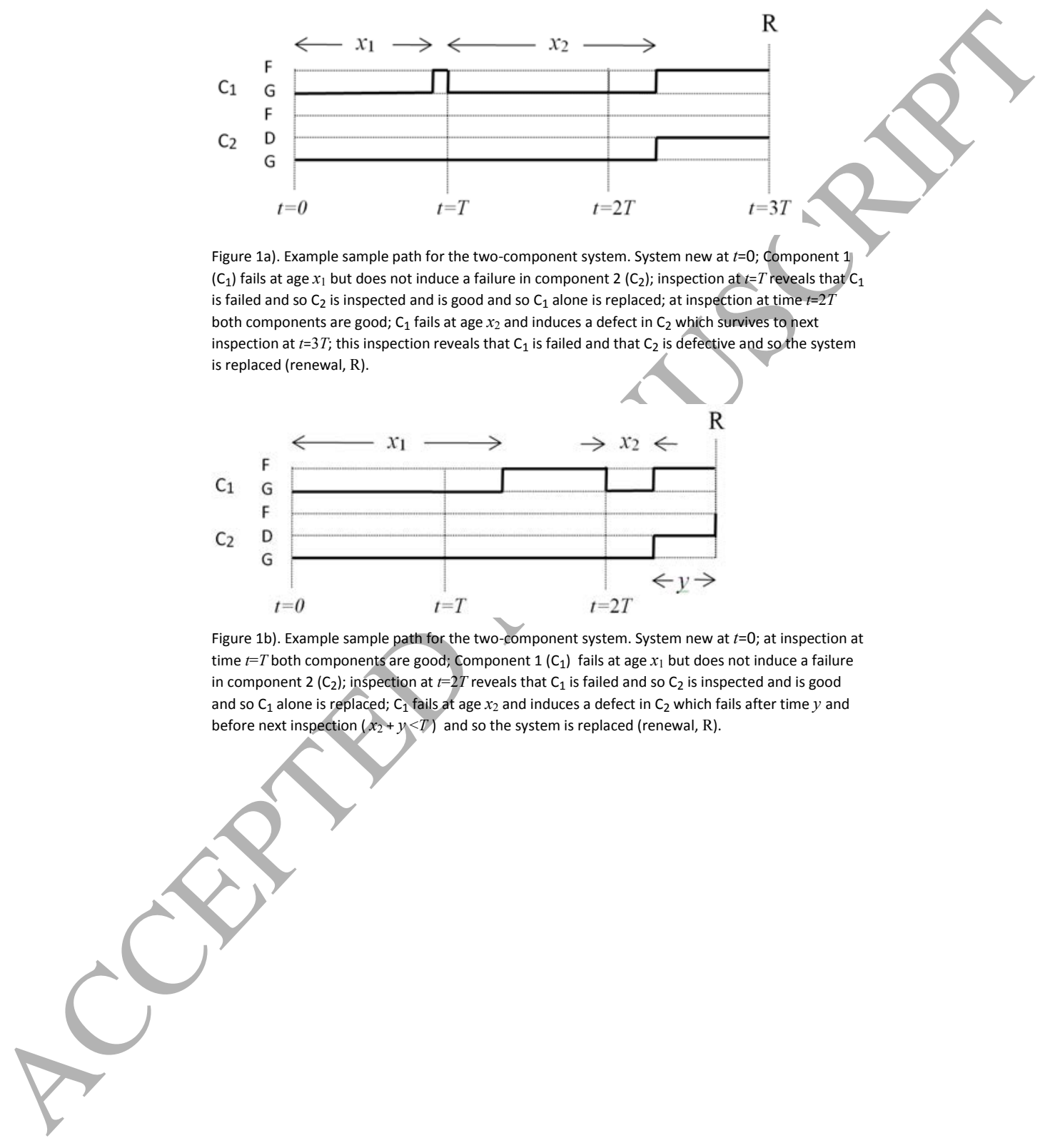




\section{Cost-rate formulation}

\subsection{Preliminaries}

When the inspection interval, $T$, is finite, there will be a sequence of inspections and replacements of component 1 prior to renewal of the system because a failure of component 1 will eventually induce a defect in component 2 which in turn wilk lead to renewal either at inspection or on failure of component 2. Thus, because the system is renewed if and only if component 2 is replaced, there may be many replacements of component 1 in a renewal cycle.

Now, for finite $T$, the number of failures of component 1 not inducing a defective state in component $2, H$, has a geometric distribution with probability function:

$$
P(H=j)=(1-p)^{j} p, j=0,1,2, \ldots,
$$

This is because $H=j$ if and only if there are $j+1$ failures of component 1 in a renewal cycle with the first $j$ not inducing a defect in component 2 and the final failure doing so. $H=0$ implies that the first replicate of component 1 induces the defective state in component 2 .

When the inspection interval is not finite, that is, there is no inspection, the asymptotic behaviour of the length of a renewal cycle and the cost function depends on whether the failure of the first replicate of component 1 induces the defective state in component 2 or not. In the former case $H=0$ and the cycle will be finite as the defective state in component 2 will eventually lead to its failure. However if the first replicate does not induce the defective state, then $(H>0)$ and the length of the renewal cycle is infinite because component 2 will never fail. This fact means we must distinguish these two cases:

- failure of the first component 1 (first replicate) induces the defective state in component 2;

- failure of the first component 1 (first replicate) does not induce the defective state in component 2

when calculating the expected length of a cycle as well as the expected cost.

In addition

$$
E[H]=\frac{1-p}{p}
$$

and conditional on the first replicate of component 1 not inducing the defective state:

$$
E[H \mid H>0]=\frac{1}{p}
$$


The number of inspections of component 1 in a cycle, $K_{1}$, takes values $0,1,2, \ldots$

Now $K_{1}=0$ if component 1 fails in the first inspection interval, induces a defect in component 2 which itself fails before the first inspection. Thus

$$
p_{0}=P\left(K_{1}=0\right)=p \int_{0}^{T} f(x)\left(\int_{0}^{T-x} g(y) d y\right) d x .
$$

Now $K_{1}=1$ in any of the three following cases:

- component 1 fails in the first inspection interval, does not induce a defect in component 2 which then survives (definitely) to the first inspection, the new component 1 then fails in the second inspection interval, and induces a defect in component 2 which fails before the subsequent inspection;

- component 1 fails in the first inspection interval, and induces a defect in component 2 which itself survives to the first inspection and is replaced;

- component 1 does not fail in the first inspection interval but fails in the second inspection interval, inducing a defect in component 2 which itself fails before the second inspection.

Therefore,

$$
\begin{aligned}
& p_{1}=P\left(K_{1}=1\right)= \\
& \int_{0}^{T} f(x)\left[p \int_{T-x}^{\infty} g(y) d y+(1-p) \int_{T}^{2 T} f(x-T) d x \int_{0}^{2 T-x} p g(y) d y\right] d x+ \\
& \int_{T}^{2 T} f(x)\left(\int_{0}^{2 T-x} p g(y) d y\right) d x= \\
& \int_{0}^{T} f(x)\left[p \int_{T-x}^{\infty} g(y) d y+(1-p) \int_{0}^{T} f(u) d u \int_{0}^{T-u} p g(y) d y\right] d x+ \\
& \int_{T}^{2 T} f(x)\left(\int_{0}^{2 T-x} p g(y) d y\right) d x= \\
& \int_{0}^{T} f(x)\left[(1-p) p_{0}+p \int_{T-x}^{\infty} g(y) d y\right] d x+p \int_{T}^{2 T} f(x)\left(\int_{0}^{2 T-x} g(y) d y\right) d x
\end{aligned}
$$

The probabilities that $K_{1}=2,3 \ldots$ can be found by reasoning in a similar manner. Thus, 


$$
\begin{aligned}
& p_{2}=P\left(K_{1}=2\right)= \\
& (1-p) p_{1} \int_{0}^{T} f(x) d x+\int_{T}^{2 T} f(x)\left((1-p) p_{0}+p \int_{2 T-x}^{\infty} g(y) d y\right) d x+ \\
& p\left(\int_{2 T}^{3 T} f(x) \int_{0}^{3 T-x} g(y) d y\right) d x
\end{aligned}
$$

and

$$
\begin{aligned}
& p_{3}=P\left(K_{1}=3\right)= \\
& (1-p) p_{2} \int_{0}^{T} f(x) d x+(1-p) p_{1} \int_{T}^{2 T} f(x) d x+ \\
& \int_{2 T}^{3 T} f(x)\left((1-p) p_{0}+p \int_{3 T-x}^{\infty} g(y) d y\right) d x+ \\
& p\left(\int_{3 T}^{4 T} f(x) \int_{0}^{4 T-x} g(y) d y\right) d x .
\end{aligned}
$$

The following general recursive formula is thus obtained for $i=1,2, \ldots$ :

$$
\begin{aligned}
& p_{i}=P\left(K_{1}=i\right)= \\
& \sum_{j=1}^{i}(1-p) p_{i-j} \int_{(j-1) T}^{j T} f(x) d x+ \\
& p\left[\int_{(i-1) T}^{i T} f(x)\left(\int_{i T-x}^{\infty} g(y) d y\right) d x+\int_{i T}^{(i+1) T} f(x)\left(\int_{0}^{(i+1) T-x} g(y) d y\right) d x\right] .
\end{aligned}
$$

If the first replicate of component 1 induces the defective state in component 2 , the previous expression is simplified and the probability that component 1 is inspected $i$ times is:

$$
\begin{aligned}
& \left.p_{i}\right|_{H=0}=P\left(K_{1}=i \mid H=0\right)= \\
& {\left[\int_{(i-1) T}^{i T} f(x)\left(\int_{i T-x}^{\infty} g(y) d y\right) d x+\int_{i T}^{(i+1) T} f(x)\left(\int_{0}^{(i+1) T-x} g(y) d y\right) d x\right] .}
\end{aligned}
$$


The following calculations allow us to obtain $E\left[K_{1}\right]$ whose expression is presented later in Proposition 1.

Let $K_{1}^{i}(i=1,2, \ldots H)$ be the number of inspections of the $i$ th replicate of component 1 conditional on the $i$ th replicate not inducing the defective state in component 2 . In addition $K_{1}^{p}$ denotes the number of inspections of the replicate that induces the defective state. In what follows $X_{i}, i=1,2, \ldots, H$ denote the times to failure of the successive components 1 that do not induce the defective state and $X_{p}$ that of the component 1 inducing the defective state. Observe that

$$
K_{1}^{i}=\left\lfloor\frac{X_{i}}{T}\right\rfloor+1, i=1,2, \ldots H
$$

and

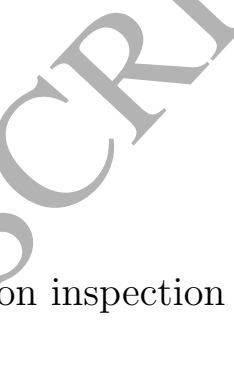

and

$$
K_{1}^{p}=\left\lfloor\frac{X_{p}}{T}\right\rfloor+1, \text { if the cycle ends on inspection }
$$

$$
K_{1}^{p}=\left\lfloor\frac{X_{p}}{T}\right\rfloor, \text { if the cycle ends on failure }
$$

where $\lfloor$.$\rfloor represents the integer part function.$

It follows that

$$
E\left[K_{1}^{i}\right]=E\left[\frac{X_{i}}{T}\right\rfloor+1=\sum_{m=0}^{\infty} \bar{F}(m T), i=1,2, \ldots H
$$

with $\bar{F}(x)$ the reliability function of component 1 .

Now let $m_{0}$ be the probability that system is renewed on inspection conditional on a replicate of component 1 inducing the defective state in component 2 . Then

$$
m_{0}=\sum_{k=1}^{\infty} \int_{(k-1) T}^{k T} f(x)\left(\int_{k T-x}^{\infty} g(y) d y\right) d x=\sum_{k=1}^{\infty} \int_{(k-1) T}^{k T} f(x) \bar{G}(k T-x) d x .
$$

with $\bar{G}$ the reliability function of $Y$.

Also let $r_{0}$ be the probability the system is renewed on failure conditional on a replicate of component 1 inducing the defective state in component 2. Then

$$
r_{0}=\sum_{k=1}^{\infty} \int_{(k-1) T}^{k T} f(x)\left(\int_{0}^{k T-x} g(y) d y\right) d x=\sum_{k=1}^{\infty} \int_{(k-1) T}^{k T} f(x) G(k T-x) d x
$$


with $G$ the distribution function of $Y$.

We can see that $r_{0}+m_{0}=1$.

Result 1: Moreover the following limits apply:

$$
\begin{aligned}
& m_{0} \rightarrow 0, \text { if } T \rightarrow \infty \\
& T m_{0} \rightarrow 0, \text { if } T \rightarrow \infty \\
& r_{0} \rightarrow 1, \text { if } T \rightarrow \infty
\end{aligned}
$$

The previous results hold since $\bar{G}(k T) \rightarrow 0$ and $T \bar{G}(k T) \rightarrow \theta$ when $T \rightarrow \infty$.

The following defines the indicator function for a cycle ending on inspection:

Definition 1 Let $A$ be the following indicator function:

$$
A=\left\{\begin{array}{l}
1, \quad \text { the cycle ends on inspection, } \quad P(I=1)=m_{0} \\
0, \quad \text { the cycle ends on failure, } \quad P(I=0)=r_{0}
\end{array}\right.
$$

with $m_{0}$ and $r_{0}$ given in (3) and (4) respectively.

From (1b) and (1c) it follows that

$$
E\left[K_{1}^{p}\right]=\left(E\left\lfloor\frac{X_{p}}{T}\right\rfloor+1\right) m_{0}+E\left\lfloor\frac{X_{p}}{T}\right\rfloor r_{0}=\sum_{m=1}^{\infty} \bar{F}(m T)+m_{0}
$$

Then, the following result applies:

\section{Proposition 1}

$$
E\left[K_{1}\right]=\frac{1-p}{p}+\frac{1}{p} \sum_{m=1}^{\infty} \bar{F}(m T)+m_{0}
$$

and

$$
E\left[K_{1} \mid H=0\right]=\sum_{m=1}^{\infty} \bar{F}(m T)+m_{0}
$$

and

$$
E\left[K_{1} \mid H>0\right]=\frac{1}{p}+\left(\frac{1}{p}+1\right) \sum_{m=1}^{\infty} \bar{F}(m T)+m_{0} .
$$


Proof: The total number of inspections of component 1 in a cycle, $K_{1}$, is given by

$$
K_{1}=\sum_{i=1}^{H} K_{1}^{i}+K_{1}^{p}
$$

with $H$ having the geometric distribution that accounts for the number of replacements of component 1 not including the one that induces the defective state. Therefore

$$
E\left[K_{1}\right]=\frac{1-p}{p} E\left[K_{1}^{i}\right]+E\left[K_{1}^{p}\right]
$$

since $E[H]=\frac{1-p}{p}$. The result in (6a) is derived from (2) and (5).

In addition $E\left[K_{1} \mid H=0\right]=E\left[K_{1}^{p}\right]$ whose expression was given in (5) and so the result in (6b) applies.

Moreover, as $E[H \mid H>0]=\frac{1}{p}$, it follows that

$$
E\left[K_{1} \mid H>0\right]=\frac{1}{p} E\left[K_{1}^{i}\right]+E\left[K_{1}^{p}\right]
$$

Then, the result in (6c) is obtained from (2) and (5) as follows

$$
\begin{aligned}
& E\left[K_{1} \mid H>0\right]=\frac{1}{p} E\left[K_{1}^{i}\right]+E\left[K_{1}^{p}\right]= \\
& =\frac{1}{p} \sum_{m=0}^{\infty} \bar{F}(m T)+\sum_{m=1}^{\infty} \bar{F}(m T)+m_{0} .
\end{aligned}
$$

Result 2: It follows that

$$
\begin{aligned}
& \lim E\left[K_{1} \mid H=0\right]=0, \text { if } T \rightarrow \infty \\
& \lim E\left[K_{1} \mid H>0\right]=\frac{1}{p}, \text { if } T \rightarrow \infty
\end{aligned}
$$

Result 2 follows from (5) and Lemma 1 in Badía et al [2] proving that $\sum_{m=1}^{\infty} \bar{F}(m T) \rightarrow 0$ when $T \rightarrow \infty$.

Note that the definition of $K_{1}$ only makes sense for $T$ finite and $K_{1}=0$ otherwise. Therefore no cost is incurred from inspection of component 1 if $T \rightarrow \infty$. The foregoing limiting result for $E\left[K_{1} \mid H>0\right]$ does not contradict this point since if $T \rightarrow \infty$ and $H>0$ 
component 2 will never fail and the cycle length is infinite. Hence, the cost per unit of time derived from $E\left[K_{1} \mid H>0\right]$ tends to 0 in the long run.

As we have indicated, there may be many replacements of component 1 before renewal of the system. When there are $i$ replacements of component 1 in a cycle $(H=i)$, the number of replicates of component 1 used in a cycle is $i+1$. The last in this sequence, the final replicate, is the one responsible for replacement of the system since it must on failure induce a defect in component 2 ; if it does not induce a defect in component 2 on failure, it cannot be the last replicate, since it will be replaced when it is found to be failed at a subsequent inspection. Many of the expressions we obtain follow as a consequence of this logic.

Now consider $K_{2}$, taking values $0,1,2, \ldots$ the number of inspections of component 2 in a renewal cycle, including the inspection at renewal if renewal occurs at inspection. If the first time that component 1 fails it induces a defect in component 2 which fails before the defect is detected, then $K_{2}=0$ and so

$$
P\left(K_{2}=0\right)=p r_{0} .
$$

Further, $K_{2}=1$ in two possible ways: the first replicate of component 1 fails in an interval and $i$ ) it induces a defect in component 2 which survives to end of interval, component 2 is inspected, and replaced or $i i$ ) it does not induce a defect in component 2 so both components are inspected but only component 1 replaced. In the latter case the second replicate of component 1 then fails in a subsequent interval and induces a defect in component 2 which fails before the following inspection. Thus, two terms arise in the following probability:

$$
\begin{aligned}
& r_{1}=P\left(K_{2}=1\right)=\sum_{k=1}^{\infty} \int_{(k-1) T}^{k T} f(x) p\left(\int_{k T-x}^{\infty} g(y) d y\right) d x+ \\
& \sum_{k=1}^{\infty} \int_{(k-1) T}^{k T} f(x)(1-p) d x \sum_{j=k}^{\infty} \int_{j T}^{(j+1) T} f(x-k T) p\left(\int_{0}^{(j+1) T-x} g(y) d y\right) d x .
\end{aligned}
$$


Equation (7) can be also expressed as follows:

$$
\begin{aligned}
& r_{1}=P\left(K_{2}=1\right)=p \sum_{k=1}^{\infty} \int_{(k-1) T}^{k T} f(x)\left(\int_{k T-x}^{\infty} g(y) d y\right) d x+ \\
& (1-p) p \sum_{r=1}^{\infty} \int_{(r-1) T}^{r T} f(x)\left(\int_{0}^{r T-x} g(y) d y\right) d x= \\
& p \sum_{k=1}^{\infty} \int_{(k-1) T}^{k T} f(x)\left(\int_{k T-x}^{\infty} g(y) d y\right) d x+(1-p) r_{0}= \\
& p m_{0}+(1-p) p_{0} .
\end{aligned}
$$

The foregoing expression relates the number of component 1 replacements and the number of inspections of component 2 in a cycle. The probability in the first term implies that component one fails, induces a defect and there is one inspection of component 2 . Then the system is renewed and the number of component 1 replacements is zero. The second term implies that component 1 fails and does not induce a defect, whereas this component 1 replacement induces a defect in component 2 which in turn fails before the defect is detected. Hence component 1 has been previously replaced once apart from the whole system replacement on component 2 failure. Therefore once a defect in component 2 is induced component 2 will be inspected one more time at most. Note that in any of these two cases $K_{1} \geq 1$, that is, component 1 can undergo several inspections before component 2 is inspected.

Expressions for $r_{2}, r_{3}$, are found using a similar argument. Thus

$$
\begin{aligned}
& r_{2}=P\left(K_{2}=2\right)=r_{1} \sum_{k=1}^{\infty}(1-p) \int_{(k-1) T}^{k T} f(x) d x= \\
& (1-p) p m_{0}+(1-p)^{2} p r_{0}
\end{aligned}
$$

and

$$
r_{i}=P\left(K_{2}=i\right)=(1-p)^{i-1} p m_{0}+(1-p)^{i} p r_{0}
$$

with $m_{0}$ and $r_{0}$ given, respectively, in (3) and (4).

The first term in (8) indicates that there are $i-1$ component 1 failures that do not induce a defect in component 2, the $i^{\text {th }}$ does and it is detected on inspection before failure of component 2 occurs. The second term is the probability of $i$ component 1 failures which do not induce a defect whereas the failure of the $i+1^{\text {th }}$ replicate of component 1 does and component 2 fails before the $i+1^{\text {th }}$ inspection. 


\section{Proposition 2}

$$
\begin{aligned}
& \qquad E\left[K_{2}\right]=\frac{1}{p} m_{0}+\frac{1-p}{p} r_{0} \\
& \text { and } E\left[K_{2} \mid H=0\right]=m_{0} \\
& \text { and } \\
& \qquad E\left[K_{2} \mid H>0\right]=\frac{1}{p} r_{0}+\frac{1+p}{p} m_{0}=\frac{1}{p}+m_{0} .
\end{aligned}
$$

Proof: The expected number of component 2 inspections in a cycle is

with $r_{i}$ in (8).

$$
E\left[K_{2}\right]=\sum_{i=1}^{\infty} \hat{i r_{i}}
$$

After straightforward calculations the result in (9a) is obtained.

This is because the number of inspections of component 2 is the same as that of component 1 replacements $\left(K_{2}=H\right)$ when component 2 fails before the induced defect is detected, and thus the cycle ends on failure. $K_{2}=H+1$ when a defect in component 2 is detected before it fails and the cycle ends at inspection.

The result in $(9 \mathrm{~b})$ holds since $H=0$ implies that the first component 1 induces the defect in component 2. If so, $K_{2}=0$ or $K_{2}=1$. The latter case occurs if the defective state is detected on inspection. The probability of this event is $m_{0} . K_{2}=0$ otherwise, that is if component 2 fails before its defective state is detected.

Moreover

$$
\begin{aligned}
& E\left[K_{2} \mid H>0\right]=E[H \mid H>0] r_{0}+(E[H \mid H>0]+1) m_{0}= \\
& \frac{1}{p} r_{0}+\frac{1+p}{p} m_{0}=\frac{1}{p}+m_{0}
\end{aligned}
$$

and thus, the result in $(9 \mathrm{c})$ is proved.

\section{Result 3:}

$$
\begin{aligned}
& \lim E\left[K_{2} \mid H=0\right]=0, \text { if } T \rightarrow \infty \\
& \lim E\left[K_{2} \mid H>0\right]=\frac{1}{p}, \text { if } T \rightarrow \infty
\end{aligned}
$$

Result 3 follows from Result 1. 
A similar comment to that for $\lim E\left[K_{1} \mid H>0\right]$ when $T \rightarrow \infty$ in Result 1 applies here for $\lim E\left[K_{2} \mid H>0\right]$. Therefore no cost is derived from inspections of component 2 if $T \rightarrow \infty$ and $H>0$ since it is a finite cost in an infinite cycle.

\subsection{Expected length and expected cost of a cycle}

A renewal occurs and a cycle ends when inspection of component 2 reveals it is defective or when component 2 fails between two consecutive inspections of component 1 . Hence the length of a cycle, $\tau$, is given by the sum of two terms:

$$
\tau=K_{1} T+Z
$$

$Z$, called the excess time, accounts for the elapsed time from the last inspection of component 1 where component 2 was not defective to failure of component $2 . Z$ is a nonnegative random variable such that $Z>0$ when the cycle ends on failure and $Z=0$ otherwise, that is, when the defective state of component 2 is revealed on inspection.

Let $q_{i}, i=0,1,2, \ldots$ be defined as follows

$$
q_{i}=\int_{i T}^{(i+1) T} f(x)\left(\int_{0}^{(i+1) T-x}(x-i T+y) g(y) d y\right) d x .
$$

$q_{i}$ accounts for the mean excess time when the replicate of component 1 that induces the defective state in component 2 fails in the interval $(i T,(i+1) T)$. The excess time does not depend on whether the replicate inducing the defective state is the first or one of the subsequent ones and hence

$$
E[Z]=E[Z \mid H=0]=E[Z \mid H>0]=\sum_{i=0}^{\infty} q_{i} .
$$

Result 4: Observe that if $T \rightarrow \infty$ then $E[Z] \rightarrow \mu_{X}+\mu_{Y}$ with $\mu_{X}=E[X]$ and $\mu_{Y}=E[Y]$.

The next result provides the conditional expected length of a cycle: 


\section{Theorem 1}

$$
\begin{aligned}
& E[\tau \mid H=0]=T\left(\sum_{m=1}^{\infty} \bar{F}(m T)+m_{0}\right)+\sum_{i=0}^{\infty} q_{i} \\
& \text { and } \\
& E[\tau \mid H>0]=T\left(\frac{1}{p} \sum_{m=0}^{\infty} \bar{F}(m T)+\sum_{m=1}^{\infty} \bar{F}(m T)+m_{0}\right)+\sum_{i=0}^{\infty} q_{i} .
\end{aligned}
$$

\section{Proof:}

Taking conditional expectations in (10), it follows that

$$
\begin{aligned}
& E[\tau \mid H=0]=T E\left[K_{1} \mid H=0\right]+E[Z \mid H=0]=T E\left[K_{1}^{p}\right]+E[Z] \\
& \text { and } \\
& E[\tau \mid H>0]=T E\left[K_{1} \mid H>0\right]+E[Z \mid H>0] .
\end{aligned}
$$

The result in (12a) follows from (6b) and (11). The expression in (12b) is obtained from (6c) and (11).

We call the time component 1 spends in the failed state the penalty time. Let $D_{i}$ be the penalty time corresponding to failure of the $i t h$ replicate of component 1 conditional that this failure does not induce a defect in component 2 . Then

$$
E\left[D_{i}\right]=\sum_{k=1}^{\infty} \int_{(k-1) T}^{k T}(k T-x) f(x) d x=T \sum_{k=0}^{\infty} R(k T)-\mu_{X} .
$$

Since, in a cycle, there are $H$ consecutive failures of component 1 that do not induce a defect in component 2 (recalling that $H$ is a geometric random variable with parameter $p)$, the total penalty time in a cycle due to such events is

$$
\sum_{i=1}^{H} D_{i}
$$

Let $D^{p}$ be the penalty time corresponding to a failure of component 1 given that this failure does induce a defect in component 2. Then

$$
E\left[D^{p}\right]=\sum_{k=1}^{\infty} \int_{(k-1) T}^{k T}\left(\int_{0}^{k T-x} y g(y) d y+\int_{k T-x}^{\infty}(k T-x) g(y) d y\right) f(x) d x .
$$


Result 5: Observe that if $T \rightarrow \infty$, then $E\left[D^{p}\right] \rightarrow \mu_{Y}$.

If $H=0$, then the first replicate of component 1 induces the defective state and there are no more replicates in a cycle. Therefore the penalty time can be only caused by this first and only replicate. When $H>0$ the mean number of replicates not inducing a defective state in component 2 is $E[H \mid H>0]=\frac{1}{p}$ and the penalty time is the sum of the penalty times incurred by all the replicates: those that do not induce the defective state and the last one which does. It follows that

$$
E[D]=E\left[\sum_{i=1}^{H} D_{i}\right]+E\left[D^{p}\right]=\frac{1-p}{p} E\left[D_{i}\right]+E\left[D^{p}\right]
$$

and

$$
E[D \mid H=0]=E\left[D^{p}\right]
$$

and

$$
E[D \mid H>0]=\frac{1}{p} E\left[D_{i}\right]+E\left[D^{p}\right] .
$$

Note that the expressions of $E\left[D_{i}\right]$ and $E\left[D^{p}\right]$ are given in (13) and (14) respectively.

A similar reasoning follows to obtain the expected cost in a renewal cycle. If $H=0$, the first and only replicate of component 1 will undergo several inspections before renewal and component 2 will be inspected once at most in the case that it does not fail before inspection. This last event occurs with probability $m_{0}$. If $H>0$ the counting extends to all the replicates: those not inducing the defective state and the last one that induces it. In addition the mean number of inspections of component 2 is $E\left[K_{2} \mid H>0\right]$.

The following result contains the expressions for the conditional expected costs in a renewal cycle:

\section{Theorem 2}

$$
E[C(\tau) \mid H=0]=c_{1} E\left[K_{1}^{p}\right]+c_{2} m_{0}+c_{d} E\left[D^{p}\right]+c_{I} m_{0}+c_{F} r_{0}
$$

nd

$E[C(\tau) \mid H>0]=$

$c_{1} E\left[K_{1} \mid H>0\right]+c_{2} E\left[K_{2} \mid H>0\right]+c_{p} E[H \mid H>0]+c_{d}\left(\frac{1}{p} E\left[D_{i}\right]+E\left[D^{p}\right]\right)+c_{I} m_{0}+c_{F} r_{0}$.

Proof: The cost of a cycle is given by

$$
C(\tau)=c_{1} K_{1}+c_{2} K_{2}+c_{d} D+c_{I} A+c_{F}(1-A)
$$


where $A$ is the indicator function given in Definition 1.

Observe that $E[A]=E[A \mid H=0]=E[A \mid H>0]=m_{0}$.

The results (16a) and (16b) are derived after taking conditional expectations in (17). The expression in (16a) follows from (9b), (15b) and $E[A \mid H=0]$. The expression in $(16 \mathrm{~b})$ is derived from $(9 \mathrm{c}),(15 \mathrm{c})$ and $E[A \mid H>0]$. The values of $E\left[K_{1} \mid H=0\right]=E\left[K_{1}^{p}\right]$ and $E\left[K_{1} \mid H>0\right]$ are given in (6b) and (6c), respectively. The expressions of $E\left[D_{i}\right]$ and $E\left[D^{p}\right]$ are given in (13) and (14).

\subsection{Cost function}

When $T$ is non-finite (no inspection), although component 1 will eventually fail, the probability that component 2 fails and the cycle ends (because failure of component 1 induces a defect in component 2) is $p$. However the probability that component 2 remains in the good state in perpetuity and the cycle length is not finite is $1-p$. In the latter case the cost-rate is simply $c_{d}$, the penalty cost-rate when component is failed. Thus, when analyzing the cost rate the cases where the first replicate of component 1 induces the defective state $(H=0)$, or not $(H \ngtr 0)$, should be taken apart. The definition of $H$ as number of failures of component 1 not inducing the defective state of component 2 applies for $T=\infty$ and the case $H>0$ means that the failure of the first and only replicate of component does not induce the defective state in component 2.

Then, when $H>0$ :

$$
Q(T \mid H=0)=\frac{E[C(\tau) \mid H=0]}{E[\tau \mid H=0]}
$$

If the first component 1 induces the defective state in component 2 and $T$ is non-finite so that there is no inspection, the cycle, $\tau \mid H=0$, is finite since it will be completed on failure of component 2 . The cost will be $c_{F}$ plus the penalty cost incurred and it follows that:

Result 6:

and

$$
\lim _{T \rightarrow \infty} E[\tau \mid H=0]=\mu_{X}+\mu_{Y}
$$

$$
\lim _{T \rightarrow \infty} E[C(\tau) \mid H=0]=c_{d} \mu_{Y}+c_{F}
$$

and

$$
\lim _{T \rightarrow \infty} Q(T \mid H=0)=\frac{c_{d} \mu_{Y}+c_{F}}{\mu_{X}+\mu_{Y}}
$$


Observe that equation (18) also follows from expressions (16a) and (12a) along with Results $1,2,3,4$ and 5 when $T \rightarrow \infty$.

Nevertheless the the foregoing limits are no longer valid if the first replicate fails without inducing the defective state in component 2.

Case $H>0$ :

$$
Q(T \mid H>0)=\frac{E[C(\tau) \mid H>0]}{E[\tau \mid H>0]}
$$

If there is no inspection then component 2 will never fail. Therefore the cycle is infinite and there will be an accumulated cost, $c_{d}$, per unit of time and the limit of the cost rate, $Q(T \mid H>0)$ is equal to $c_{d}$. Thus

\section{Result 7:}

$$
\lim _{T \rightarrow \infty} E[\tau \mid H>0]=\infty
$$

and

$$
\lim _{T \rightarrow \infty} Q(T \mid H>0)=c_{d}
$$

Observe that (19) also follows from (16b) and (12b) along with Results 1, 2, 3, 4 and 5 when $T \rightarrow \infty$.

Hence the cost function turns out to be

$$
Q(T)=(1-p) \frac{E[C(\tau) \mid H>0]}{E[\tau \mid H>0]}+p \frac{E[C(\tau) \mid H=0]}{E[\tau \mid H=0]} .
$$

Result 8: The following limits apply

$$
\begin{aligned}
& \text { and } \lim _{T \rightarrow 0} Q(T)=\infty \\
& \lim _{T \rightarrow \infty} Q(T)=(1-p) c_{d}+p \frac{c_{d} \mu_{Y}+c_{F}}{\mu_{X}+\mu_{Y}}=c_{d}+p \frac{c_{F}-c_{d} \mu_{X}}{\mu_{X}+\mu_{Y}} .
\end{aligned}
$$

The previous considerations about the behaviour of the cost function when the failure of the first replicate induces or not the defective state lead to the expression of the result when $T \rightarrow \infty$. The limit when $T \rightarrow 0$ is obtained as follows: 
Badía et al ([2]) prove that $\lim _{T \rightarrow 0} \sum_{m=1}^{\infty} \bar{F}(m T)=\infty$ and $\lim _{T \rightarrow 0} T \sum_{m=1}^{\infty} \bar{F}(m T)=$ $\mu_{X}$. Hence $\lim _{T \rightarrow 0} \sum_{m=0}^{\infty} \bar{F}(m T)=\infty$ and $\lim _{T \rightarrow 0} T \sum_{m=0}^{\infty} \bar{F}(m T)=\mu_{X}$. Thus, $\lim _{T \rightarrow 0} Q(T)=$ $\infty$.

Result 9: Concerning the existence of a finite optimum policy, $T^{\star}$, there must exist a finite $T$

$$
Q(T)<c_{d}+p \frac{c_{F}-c_{d} \mu_{X}}{\mu_{X}+\mu_{Y}} .
$$

If there is no such $T$, then $T^{\star}=\infty$.

Badía et al. ([2]-[5] and Berrade et al. [8] provide conditions ensuring the existence of a finite $T^{\star}$ for inspection and maintenance of one-component or multiple-component systems. However none of the foregoing references considered conditional inspection. Thus a similar study does not apply either for the cost function here or its derivative which is also difficult to deal with. Although the graphs of $Q(T)$ show a convex function in the particular cases analyzed, a general result in this sense is not presented. The solutions in section 5 (next) are obtained using the optimisation package in Maple. The solutions were in addition confirmed using the Maple add-on optimisation package DirectSearch. In all cases the cost function is convex over a finite horizon that covers typical system lifetime. Note that a very large inspection interval does not provide its intended purpose (to prevent component 2 failure). Therefore, our numerical results provide optimal solutions at least in the region of interest of the decision variable.

Note that, following the consideration above and when the condition stated in Result 9 holds, we do not present a closed form solution for $T^{\star}$ and only numerical solutions are provided.

\section{Case study results}

In the numerical study that follows we assume that $X_{1}, X_{2}, \ldots$ (time to failure of component 1) are i.i.d. exponentially distributed with mean $a$. The delay time for component $2 Y$ is Weibull distributed with shape parameter 2 and scale parameter $c$. The cost of replacement of component 1 is the reference value, thus $c_{p}=1$. In particular $c_{1}=0.005$, $c_{2}=0.05, c_{I}=2.5, c_{F}=25$ and $c_{d}=0.035$ in the base case. 
Table1: effect of changing the mean time to failure of component 1 and interaction with $\boldsymbol{p}$.

\begin{tabular}{|c|c|c|c|c|c|c|c|c|c|c|c|c|}
\hline Case & $\boldsymbol{a}$ & $\boldsymbol{c}$ & $\boldsymbol{p}$ & $\boldsymbol{c}_{\boldsymbol{I}}$ & $\boldsymbol{c}_{\boldsymbol{2}}$ & $\boldsymbol{c}_{\boldsymbol{d}}$ & $\boldsymbol{c}_{\boldsymbol{I}}$ & $\boldsymbol{c}_{\boldsymbol{F}}$ & $\boldsymbol{T}^{*}$ & $\boldsymbol{Q}^{*}$ & $\boldsymbol{Q}(\boldsymbol{\infty})$ & $\boldsymbol{\%}$ \\
\hline 1 & $\mathbf{1 5}$ & 5 & 0.1 & 0.005 & 0.05 & 0.035 & 2.5 & 25 & 0.987 & 0.095 & 0.160 & 41 \\
\hline 2 & $\mathbf{1 2}$ & 5 & 0.1 & 0.005 & 0.05 & 0.035 & 2.5 & 25 & 0.966 & 0.117 & 0.185 & 36.7 \\
\hline 3 & $\mathbf{1 0}$ & 5 & 0.1 & 0.005 & 0.05 & 0.035 & 2.5 & 25 & 0.965 & 0.138 & 0.201 & 68.7 \\
\hline 4 & $\mathbf{8}$ & 5 & 0.1 & 0.005 & 0.05 & 0.035 & 2.5 & 25 & $\mathbf{0 . 9 9 0}$ & 0.169 & 0.234 & 27.8 \\
\hline 5 & $\mathbf{5}$ & 5 & 0.1 & 0.005 & 0.05 & 0.035 & 2.5 & 25 & 1.192 & 0.258 & 0.298 & 13.4 \\
\hline 6 & $\mathbf{1 5}$ & 5 & 0.2 & 0.005 & 0.05 & 0.035 & 2.5 & 25 & 0.791 & 0.112 & 0.287 & 61 \\
\hline 7 & $\mathbf{1 2}$ & 5 & 0.2 & 0.005 & 0.05 & 0.035 & 2.5 & 25 & 0.766 & 0.138 & 0.334 & 58.7 \\
\hline 8 & $\mathbf{1 0}$ & 5 & 0.2 & 0.005 & 0.05 & 0.035 & 2.5 & 25 & 0.755 & 0.163 & 0.377 & 56.8 \\
\hline 9 & $\mathbf{8}$ & 5 & 0.2 & 0.005 & 0.05 & 0.035 & 2.5 & 25 & $\mathbf{0 . 7 5 6}$ & 0.200 & 0.433 & 53.8 \\
\hline 10 & $\mathbf{5}$ & 5 & 0.2 & 0.005 & 0.05 & 0.035 & 2.5 & 25 & 0.851 & 0.308 & 0.561 & 45.1 \\
\hline 11 & $\mathbf{1 5}$ & 5 & $\mathbf{0 . 4}$ & 0.005 & 0.05 & 0.035 & 2.5 & 25 & 0.656 & 0.138 & 0.539 & 74.4 \\
\hline 12 & $\mathbf{1 2}$ & 5 & 0.4 & 0.005 & 0.05 & 0.035 & 2.5 & 25 & 0.632 & 0.169 & 0.633 & 73.3 \\
\hline 13 & $\mathbf{1 0}$ & 5 & 0.4 & 0.005 & 0.05 & 0.035 & 2.5 & 25 & 0.619 & 0.200 & 0.718 & 72.1 \\
\hline 14 & $\mathbf{8}$ & 5 & 0.4 & 0.005 & 0.05 & 0.035 & 2.5 & 25 & 0.614 & 0.247 & 0.830 & 70.2 \\
\hline 15 & $\mathbf{5}$ & 5 & 0.4 & 0.005 & 0.05 & 0.035 & 2.5 & 25 & $\mathbf{0 . 6 6 5}$ & 0.381 & 1.088 & 65 \\
\hline 16 & $\mathbf{1 5}$ & 5 & $\mathbf{0 . 8}$ & 0.005 & 0.05 & 0.035 & 2.5 & 25 & 0.566 & 0.171 & 1.043 & 83.6 \\
\hline 17 & $\mathbf{1 2}$ & 5 & 0.8 & 0.005 & 0.05 & 0.035 & 2.5 & 25 & 0.543 & 0.211 & 1.232 & 82.9 \\
\hline 18 & $\mathbf{1 0}$ & 5 & 0.8 & 0.005 & 0.05 & 0.035 & 2.5 & 25 & 0.531 & 0.250 & 1.401 & 82.2 \\
\hline 19 & $\mathbf{8}$ & 5 & 0.8 & 0.005 & 0.05 & 0.035 & 2.5 & 25 & 0.525 & 0.308 & 1.626 & 81.1 \\
\hline 20 & $\mathbf{5}$ & 5 & 0.8 & 0.005 & 0.05 & 0.035 & 2.5 & 25 & $\mathbf{0 . 5 6 0}$ & 0.478 & 2.141 & 77.7 \\
\hline
\end{tabular}


Table 1 analyzes the effect on the optimum policy of changing the mean time to failure of component 1 and the way that this pattern is affected by $p$. The comparison between inspection versus no inspection $\left(T^{\star}=\infty\right)$ constitutes an important point and hence the cost in the latter, $Q(\infty)$, is included along with how much better (in percentage terms) is the former. The results point to:

1.a) The greater the probability of inducing a failure, $p$, the more advantageous inspection is (in term of cost) compared to no inspection.

1.b) As the probability of inducing a failure increases, $T^{\star}$ decreases. The interest of preventing the failure is more evident.

1.c) The advantage of inspection decreases as the mean time to failure of component 1 decreases. In fact $T^{\star}$ is not monotonic with a. Although inspection seems to be the preferable option if component 1 fails very often, it is not worth increasing the inspection frequency without limit. There is a kind of "threshold" in the parameter $a$ so that $T^{\star}$ starts to increase when $a$ is below this threshold. If the rate of component 1 failures exceeds a particular limit a more reliable component 1 with a lower failure rate would increase the economic benefit derived from inspection. This behavior depends also on $p$ : the greater $p$, the lower the threshold. The larger $p$, the more beneficial is frequent inspection. 
Table2: effect of changing the delay time of component 2 and interaction with $\boldsymbol{p}$.

\begin{tabular}{|c|c|c|c|c|c|c|c|c|c|c|c|c|}
\hline Case & $\boldsymbol{a}$ & $\boldsymbol{c}$ & $\boldsymbol{p}$ & $\boldsymbol{c}_{\boldsymbol{d}}$ & $\boldsymbol{c}_{\boldsymbol{I}}$ & $\boldsymbol{c}_{\boldsymbol{F}}$ & $\boldsymbol{T}^{*}$ & $\boldsymbol{E}\left[\boldsymbol{K}_{\boldsymbol{I}}\right]$ & $\boldsymbol{E}\left[\boldsymbol{K}_{\boldsymbol{2}}\right]$ & $\boldsymbol{Q}^{*}$ & $\boldsymbol{Q}(\boldsymbol{\infty})$ & $\boldsymbol{\%}$ \\
\hline 1 & 15 & 5 & 0.1 & 0.035 & 2.5 & 25 & 0.987 & 157.02 & 9.99 & 0.095 & 0.161 & 41 \\
\hline 2 & 15 & $\mathbf{8}$ & 0.1 & 0.035 & 2.5 & 25 & 1.475 & 106.77 & 9.99 & 0.092 & 0.146 & 37 \\
\hline 3 & 15 & $\mathbf{1 0}$ & 0.1 & 0.035 & 2.5 & 25 & 1.833 & 86.92 & 9.99 & 0.091 & 0.138 & 34.1 \\
\hline 4 & 15 & $\mathbf{1 2}$ & 0.1 & 0.035 & 2.5 & 25 & 2.230 & 72.38 & 9.99 & 0.090 & 0.130 & 30.8 \\
\hline 5 & 15 & $\mathbf{5}$ & $\mathbf{0 . 2}$ & 0.035 & 2.5 & 25 & 0.791 & 97.33 & 4.99 & 0.112 & 0.287 & 61 \\
\hline 6 & 15 & $\mathbf{8}$ & 0.2 & 0.035 & 2.5 & 25 & 1.168 & 66.74 & 4.99 & 0.108 & 0.257 & 58 \\
\hline 7 & 15 & $\mathbf{1 0}$ & 0.2 & 0.035 & 2.5 & 25 & 1.436 & 54.76 & 4.99 & 0.107 & 0.240 & 55.4 \\
\hline 8 & 15 & $\mathbf{1 2}$ & 0.2 & 0.035 & 2.5 & 25 & 1.727 & 45.97 & 4.99 & 0.106 & 0.226 & 53.1 \\
\hline 9 & 15 & $\mathbf{5}$ & $\mathbf{0 . 4}$ & 0.035 & 2.5 & 25 & 0.656 & 58.42 & 2.49 & 0.138 & 0.539 & 74.4 \\
\hline 10 & 15 & $\mathbf{8}$ & 0.4 & 0.035 & 2.5 & 25 & 0.964 & 40.16 & 2.50 & 0.133 & 0.478 & 72.2 \\
\hline 11 & 15 & $\mathbf{1 0}$ & 0.4 & 0.035 & 2.5 & 25 & 1.181 & 33.01 & 2.50 & 0.132 & 0.445 & 70.3 \\
\hline 12 & 15 & $\mathbf{1 2}$ & 0.4 & 0.035 & 2.5 & 25 & 1.416 & 27.75 & 2.50 & 0.130 & 0.417 & 68.8 \\
\hline 13 & 15 & $\mathbf{2}$ & $\mathbf{0 . 8}$ & 0.035 & 2.5 & 25 & 0.291 & 65.05 & 1.24 & 0.184 & 1.202 & 84.7 \\
\hline 14 & 15 & $\mathbf{5}$ & 0.8 & 0.035 & 2.5 & 25 & 0.566 & 33.75 & 1.25 & 0.171 & 1.043 & 83.6 \\
\hline 15 & 15 & $\mathbf{8}$ & 0.8 & 0.035 & 2.5 & 25 & 0.831 & 23.19 & 1.25 & 0.166 & 0.921 & 82 \\
\hline 16 & 15 & $\mathbf{1 0}$ & 0.8 & 0.035 & 2.5 & 25 & 1.018 & 19.05 & 1.25 & 0.164 & 0.856 & 80.8 \\
\hline 17 & 15 & $\mathbf{1 2}$ & 0.8 & 0.035 & 2.5 & 25 & 1.221 & 15.99 & 1.25 & 0.163 & 0.799 & 79.6 \\
\hline
\end{tabular}


The effect on the optimum policy of changes in the delay time are presented in Table

2. The most relevant results are:

2.a) $T^{\star}$ is monotonic with $c$. On the contrary to $a$, decreasing $c$, that is decreasing the mean delay time of component 2 , makes inspection more profitable. When the time component 2 spends in the defective state is shorter, the maintainer should prevent its failure by inspecting component 1 more frequently, because of the conditional nature of the inspection policy (inspect component 2 if and only if component 1 is failed) and the relatively low cost of inspection of component 1 .

2.b) Changes in the mean delay time affect the expected number of inspections of component 1 per renewal cycle, $E\left[K_{1}\right]$, but not that of component $2, E\left[K_{2}\right]$. When the mean delay time decreases, $E\left[K_{1}\right]$ increases whereas $E\left[K_{2}\right]$ remains stable.

Moreover $E\left[K_{2}\right]$ is close to $1 / p$ and this is so because $K_{2}=H+1$ when a cycle ends on inspection, that is when component 2 is found to be defective before it fails. This point was explained after Proposition 2. Since the purpose of inspection is to detect the defective states that precede failures, it is reasonable that the numerical results lead to $E\left[K_{2}\right]=E[H+1]=\frac{1}{p}$.

These results show that increasing the number and frequency of cheap inspections of component 1 can prevent the failure without increasing the frequency of expensive inspections of component 2. It can be observed that in this way the optimum cost increases just a small amount.

2.c) Changes in $p$ affect both $E\left[K_{1}\right]$ and $E\left[K_{2}\right]$. When $p$ increases, then $E\left[K_{1}\right]$ and $E\left[K_{2}\right]$ decrease since the higher the probability of inducing a defective state the smaller the length of the cycle. Moreover, the optimum cost increases significantly.

The cases in Tables 1 and 2 seem to indicate that when the cost of replacing on inspection is significantly lower than that of replacing on failure there exists a finite inspection policy no matter what are the values of the other parameters. Even for very low values of the mean time to failure of component 1 or mean delay time of component 2 , inspection tends to be beneficial.

Table 3 analyzes the behaviour of the optimum policy under changes to the penalty cost-rate of component $1, c_{d}$, or the cost of system replacement when component 2 is defective (preventive system replacement), $c_{I}$, as well as its interaction with $p$.

3.a) When $c_{d}$ is larger, inspection is more frequent and the more beneficial compared to no inspection. 
3.b) As $p$ increases, $T^{\star}$ decreases and thus the interest of preventing the failure of component 2 is more evident.

3.c) Inspection is less beneficial as the cost of preventive system replacement increases. There appears to be a limit for $c_{I}$ so that beyond this inspection is no longer optimum. The smaller is $p$, the smaller is this limit. Moreover this limit is also affected by the value of $c_{d}$, so that this limit increases as $c_{d}$ increases. 
Table3: effect of changing $\boldsymbol{c}_{\boldsymbol{d}} \boldsymbol{c}_{I}$ and interaction with $\boldsymbol{p}$.

\begin{tabular}{|c|c|c|c|c|c|c|c|c|c|c|c|c|}
\hline Case & $\boldsymbol{a}$ & $\boldsymbol{c}$ & $\boldsymbol{p}$ & $\boldsymbol{c}_{\boldsymbol{1}}$ & $\boldsymbol{c}_{\boldsymbol{2}}$ & $\boldsymbol{c}_{\boldsymbol{d}}$ & $\boldsymbol{c}_{\boldsymbol{I}}$ & $\boldsymbol{c}_{\boldsymbol{F}}$ & $\boldsymbol{T}^{*}$ & $\boldsymbol{Q}$ & $\boldsymbol{Q}(\boldsymbol{)})$ & $\boldsymbol{\%}$ \\
\hline 1 & 15 & 8 & 0.1 & 0.005 & 0.05 & 0.035 & 2.5 & 25 & 1.475 & 0.092 & 0.146 & 37 \\
\hline 2 & 15 & 8 & 0.1 & 0.005 & 0.05 & $\mathbf{0 . 0 6 5}$ & 2.5 & 25 & 1.325 & 0.093 & 0.174 & 46.6 \\
\hline 3 & 15 & 8 & 0.1 & 0.005 & 0.05 & $\mathbf{0 . 0 8}$ & 2.5 & 25 & 1.259 & 0.094 & 0.188 & 50 \\
\hline 4 & 15 & 8 & 0.1 & 0.005 & 0.05 & $\mathbf{0 . 2}$ & 2.5 & 25 & 0.907 & 0.098 & 0.300 & 67.3 \\
\hline 5 & 15 & 8 & 0.4 & 0.005 & 0.05 & $\mathbf{0 . 0 3 5}$ & 2.5 & 25 & 0.964 & 0.133 & 0.478 & 72.2 \\
\hline 6 & 15 & 8 & 0.4 & 0.005 & 0.05 & $\mathbf{0 . 0 6 5}$ & 2.5 & 25 & 0.917 & 0.134 & 0.500 & 73.2 \\
\hline 7 & 15 & 8 & 0.4 & 0.005 & 0.05 & $\mathbf{0 . 0 8}$ & 2.5 & 25 & 0.895 & 0.135 & 0.511 & 73.6 \\
\hline 8 & 15 & 8 & 0.4 & 0.005 & 0.05 & $\mathbf{0 . 2}$ & 2.5 & 25 & 0.751 & 0.138 & 0.598 & 76.9 \\
\hline 9 & 15 & 8 & $\mathbf{0 . 1}$ & 0.005 & 0.05 & 0.035 & $\mathbf{5}$ & 25 & 1.770 & 0.120 & 0.146 & 17.8 \\
\hline 10 & 15 & 8 & 0.1 & 0.005 & 0.05 & 0.035 & $\mathbf{6}$ & 25 & 1.925 & 0.132 & 0.146 & 9.6 \\
\hline 11 & 15 & 8 & 0.1 & 0.005 & 0.05 & 0.035 & $\mathbf{7}$ & 25 & 2.107 & 0.143 & 0.146 & 2.1 \\
\hline 12 & 15 & 8 & 0.1 & 0.005 & 0.05 & 0.035 & $\mathbf{8}$ & 25 & 2.323 & 0.154 & $\mathbf{0 . 1 4 6}$ & $\mathbf{- 5 . 5}$ \\
\hline 13 & 15 & 8 & 0.1 & 0.005 & 0.05 & 0.035 & $\mathbf{9}$ & 25 & 2.581 & 0.164 & $\mathbf{0 . 1 4 6}$ & $\mathbf{- 1 2 . 3}$ \\
\hline 14 & 15 & 8 & $\mathbf{0 . 4}$ & 0.005 & 0.05 & 0.035 & $\mathbf{5}$ & 25 & 1.219 & 0.224 & 0.478 & 53.1 \\
\hline 15 & 15 & 8 & 0.4 & 0.005 & 0.05 & 0.035 & $\mathbf{9}$ & 25 & 1.968 & 0.368 & 0.478 & 23 \\
\hline 16 & 15 & 8 & 0.4 & 0.005 & 0.05 & 0.035 & $\mathbf{1 0}$ & 25 & 2.253 & 0.402 & 0.478 & 15.5 \\
\hline 17 & 15 & 8 & 0.4 & 0.005 & 0.05 & 0.035 & $\mathbf{1 2}$ & 25 & 2.300 & 0.470 & 0.478 & 1.7 \\
\hline 18 & 15 & 8 & 0.4 & 0.005 & 0.05 & 0.035 & $\mathbf{1 3}$ & 25 & 3.482 & 0.502 & $\mathbf{0 . 4 7 8}$ & $\mathbf{- 5}$ \\
\hline 19 & 15 & 8 & 0.8 & 0.005 & 0.05 & 0.035 & 13 & 25 & 3.311 & 0.744 & 0.921 & 19.2 \\
\hline 20 & 15 & 8 & 0.8 & 0.005 & 0.05 & 0.035 & $\mathbf{1 4}$ & 25 & 3.868 & 0.794 & 0.921 & 13.8 \\
\hline 21 & 15 & 8 & 0.8 & 0.005 & 0.05 & 0.035 & $\mathbf{1 6}$ & 25 & 5.405 & 0.885 & 0.921 & 3.9 \\
\hline 22 & 15 & 8 & 0.8 & 0.005 & 0.05 & 0.035 & $\mathbf{1 7}$ & 25 & 6.531 & 0.927 & $\mathbf{0 . 9 2 1}$ & $\mathbf{- 0 . 7}$ \\
\hline 23 & 15 & 8 & 0.8 & 0.005 & 0.05 & 0.2 & 17 & 25 & 5.381 & 0.954 & 0.997 & 4.3 \\
\hline 24 & 15 & 8 & 0.8 & 0.005 & 0.05 & 0.2 & $\mathbf{1 8}$ & 25 & 6.659 & 0.995 & 0.997 & 0.2 \\
\hline 25 & 15 & 8 & 0.8 & 0.005 & 0.05 & 0.2 & $\mathbf{1 9}$ & 25 & 8.595 & 1.030 & $\mathbf{0 . 9 9 7}$ & $\mathbf{- 3 . 3}$ \\
\hline & & & & & & & & & & & & \\
\hline
\end{tabular}


The results in Table 3 show that when the difference between preventive and corrective system replacement (that is between $c_{I}$ and $c_{F}$ ) is not so large then $T^{\star}$ is infinite. Moreover a range of behaviours in $T^{\star}$ exist. In Figure $2 p=0.1$ and $c_{d}=0.2$ lead to an infinite $T^{\star}$. An infinite $T^{\star}$ can change to a finite $T^{\star}$ if $c_{d}$ increases as this is the cost rate if component 1 remains eternally failed or if $p$ is sufficiently small.

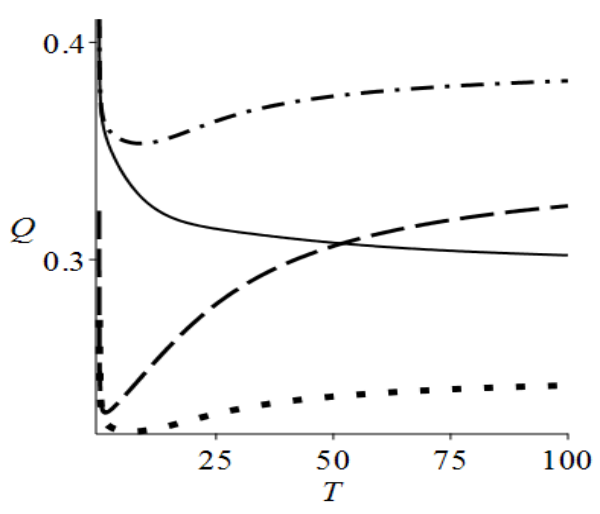

Figure 2a: cost -rate as a function of inspection interval for $p=0.05, c_{d}=0.2(\ldots.) ; p=0.1, c_{d}=0.2(-)$; $p=0.05, c_{d}=0.3(--) ; p=0.1, c_{d}=0.3(-\cdots)$

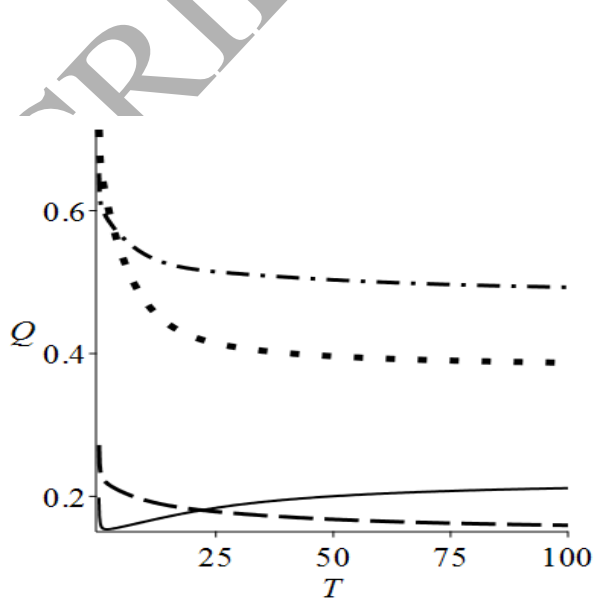

Figure $2 \mathrm{~b}$ : cost -rate as a function of inspection interval for $a=5, p=0.05(\ldots),. c_{d}=0.2 ; p=0.025, c_{d}=0.2(-)$; $p=0.05, c_{d}=0.1(---) ; p=0.2 c_{d}=0.3(-\cdot-)$

For practical purposes it would be interesting to infer a critical value for the difference between $c_{I}$ and $c_{F}$ at which $T^{*}$ becomes infinite. However it appears extremely difficult to derive this critical value theoretically given the complexity of the cost function and its derivative. Simulation appears to be the only way to address this point.

Figures $2 \mathrm{a}$ ) and $2 \mathrm{~b}$ ) show $Q(T)$ as a function of $T$ for different values of the parameters. The graphs in Figure 2b) correspond to the same cases in Figure 2a) but modifying one parameter. The case corresponding to $T^{\star}=\infty$, the solid line in $2 \mathrm{a}$ ), now gives a finite $T$ 
in $2 \mathrm{~b}$ ) by reducing $p$. Reducing the mean time to failure from $a=15$ to $a=5$, or $c_{d}$ from 0.3 to 0.1 or, increasing $p$ from 0.1 to 0.2 changes the optimum "finite" policies of Figure $2 \mathrm{a})$ to the optimum "infinite" policies shown in Figure $2 \mathrm{~b}$ ). This latter case constitutes the opposite behaviour to that observed in Tables 1, 2 and 3 .

We observed in Table 1 that when the mean time to failure of component 1 , a, decreases, inspection begins to increase when $a$ is below a certain threshold, although $T^{\star}$ is always finite provided the preventive system replacement cost substantially less than the corrective system replacement cost. When these costs are not that different, reducing $a$ increases the optimum policy in a manner that $T^{\star}$ changes from finite to infinite. Nevertheless the behavior of $T^{\star}$ when $p$ increases is contrary to that observed in Table 1. Increasing $p$ transforms a finite optimum into an infinite one, although this situation can be reversed by increasing $c_{d}$ again.

The results as they relate to the case study make sense from an engineering point of view. First, if the cost of preventive replacement of the mobile (major) part, when it is defective following a failure in the static (minor) part, is not significantly less than the cost of its corrective replacement (on failure), then there is no benefit from inspection. Further, the effectiveness of inspection is related to the penalty cost of the failure of the static part $\left(c_{d}\right)$ : if failure of the static part does not influence the quality of the rebarmesh or the cost of production (through e.g. re-work), then it is not necessary to know the state of this part, and so there is no benefit from inspection. The implication is that the maintenance manager should seek to reduce the cost of preventive replacement of the mobile part (on inspection) by making sure the resources to replace both parts are available at inspection, so that if the mobile part is defective its replacement will be prompt and interfere less with the production schedule.

Concerning the parameter values assumed in this section, it is important to note that obtaining appropriate estimates is crucial for the maintenance to be effective. Quite often historical failure data as well as results at inspection are not available due to confidentiality reasons, or they are insufficient. In addition the delay time, $Y$, is not directly measurable in most of cases. Therefore, in practice estimation of these parameters would proceed subjectively, using expert opinion (Wang [34]). Si et al. [30] present a review of developments for estimating the remaining useful life of a system at any given time.

\section{Conclusions}

We propose an inspection policy for a two component system. Component 2 is the critical component and the main target of maintenance to avoid failures. Inspection and replacement of component 1 is cheap and straightforward whereas inspection of component 2 
requires more effort and its replacement incurs a higher cost. The failure of component 1 may induce a defective state of component 2 which in turn leads to its failure. The implication is that there can be many replacements of component 1 before component 2 is renewed. The policy we propose constitutes an opportunity-based inspection when failures of component 1 trigger inspections of component 2 and its maintenance if required. In addition the policy can be viewed as a new extension of the delay time model from oneto two-component systems. Failures of component 1 can be interpreted as defect arrivals to the system that may or may not produce a defective state that in turn leads to system failure. The model development is motivated by a case study of an industrial process for cutting rebar mesh. The conditional policy takes into account the special relevance of the component 2 and the interest to prevent it from failing.

When analyzing the existence of a finite optimum policy, the results show that inspection is beneficial, that is $T^{\star}$ is finite, when the preventive system replacement cost is significantly lower than the corrective system replacement cost. In this case the values of the other parameters have little influence upon the existence of a finite inspection interval. In addition, as failures of component 1 are an indicator of possible failures of component 2 , the mean number of inspections of the latter per renewal is robust to a decrease in the mean delay time of component 2 , wherein more frequent inspection of component 1 is recommended to avoid failures of component 2. Inspections of component 1 are lowcost in contrast to those of component/2, therefore an indirect but reliable indicator of unrevealed defects in a main system emerges as a beneficial policy when inspection of the main system is very expensive. An increasing probability of inducing failure also leads to an effective policy with more frequent inspections of the low-cost type.

Nevertheless when the preventive and corrective system replacement costs are not so different, the effect of other parameters, especially the penalty cost-rate of component 1 , $c_{d}$, and the probability of inducing failure, $p$, on the existence of a finite optimum policy is more profound. Then, a slight change in any of these can turn a finite policy into an infinite one or vice versa.

The model in this paper excludes any type of failure of component 2 other than those induced by component 1 . However a natural extension emerges by assuming that component 2 additionally experiences its own failures that "compete" with induced failures. The analysis of such a model is a topic for further research.

\section{Acknowledgements}

The work of M.D. Berrade has been supported by the Spanish Ministry of Economy and Competitiveness under Project MTM2015-63978-P. The work of Cristiano Cavalcante has 
been supported by CNPq (Brazilian Research Council). The authors thank Guilherme Arthur Brunet Monteiro of GERDAU, Brazil, for discussions regarding the billet production of rebarmesh. The authors acknowledge the valuable comments of the anonymous reviewers.

\section{References}

[1] Ascher, H.E. and Feingold H. (1984).Repairable Systems Reliability. New York: Marcel Dekker.

[2] Badía, F.G; Berrade, M.D. and Campos, C.A. (2001). Optimization of inspection intervals based on cost. Journal of Applied Probability, 38, 872-881.

[3] Badía, F.G; Berrade, M.D. and Campos, C.A. (2002). Maintenance policy for multivariate standby/operating units. Applied Stochastic Models in Business and Industry, 18 (2), 147-155.

[4] Badía, F.G; Berrade, M.D. and Campos, C.A. (2002). Optimal inspection and preventive maintenance of units with revealed and unrevealed failures Reliability Engineering and System Safety, 78 (2), 157-163.

[5] Badía, F.G; Berrade, M.D. and Campos, C.A. (2002). Optimum inspection under competing risks with proportional hazards. Applied Stochastic Models in Business and Industry, 18 (4), 369-379.

[6] Barros, A.; Berenguer, C. and \& Grall, A. (2006). A maintenance policy for two-unit parallel systems based on imperfect monitoring information. Reliability Engineering and System Safety, 91, 131-136.

[7] Berrade, M.D.; Scarf, P.A. and Cavalcante, C.A.V. (2014). A bivariate delay-time model. Reliability and Risk Analysis: Beyond the Horizon. Editors: R.D.J.M. Steenbergen,, P.H.A.J.M. van Gelder, S.Miraglia, and A.C.W.M. Vrouwenvelder. CRC Press-Taylor \& Francis Group, 1023-1026.

[8] Berrade, M.D.; Scarf, P.A. and Cavalcante, C.A.V. (2015). Some Insights Into the Effect of Maintenance Quality for a Protection System. IEEE Transactions on Reliability, 64 (2), 661-672.

[9] Cho, D.I.; Parlar, M. (1991). A survey of maintenance models for multi-unit systems. European Journal of Operational Research, 51, 1-23. 
[10] Christer, A.H. (1987). Delay-time model of reliability of equipment subject to inspection monitoring. Journal of the Operational Research Society, 38(4), 329-34.

[11] Deloitte (2006). The service revolution in global manufacturing industies. Deloitte Research. New York.

[12] Dekker, R.; Wildeman, R.E. and Van Der Duyn Schouten, F.A. (1997). A Review of Multi-Component Maintenance Models with Economic Dependence. Mathematical Methods of Operations Research, 45, 411-435.

[13] Golmakani; H.R., Moakedi, H. (2012). Periodic inspection optimization model for a two-component repairable system with failure interaction. Computers \& Industrial Engineering, 63, 540-545.

[14] Heng, A.; Zhang, S.;Tan A.C.C. and Mathew, J. (2009). Rotating machinery prognostics: State of the art, challenges and opportunities. Mechanical Systems and Signal Processing, 23, 724-739.

[15] Lai, M., Chen, Y. (2006). Optimal periodic replacement policy for a two-unit system with failure rate interaction. International Journal of Advanced Manufacturing Technology, 29, 367-371.

[16] Liu, B.; Wu, S.; Xie, M. and Kuo, W. (2017). A condition-based maintenance policy for degrading systems with age and state-dependent operating cost. European Journal of Operational Research, 263, 879-887.

[17] Lu, X.; Wang, W., Yang, H.; Zuod, M.J. \& Zhoua, D. (2012). Optimizing the Periodic Inspection Interval for a 1-out-of-2 Cold Standby System Using the Delay-Time Concept. Quality and Reliability Engineering International, 28, 648-662.

[18] Murthy, D.N.P. \& Nguyen, D.G., (1985). Study of two-component systems with failure interactions. Naval Res.Logist.Quart., 32, 239-247.

[19] Nakagawa, T. (1993). Optimal replacement policies for a two-unit system with failure interactions. Recherche opérationnelle/Operations research, 27, 427-438.

[20] Nicolai, R.P.; Dekker, R. (2007). Optimal Maintenance of Multi-Component Systems: A Review. Complex System Maintenance Handbook. London: Springer 263-86.

[21] Olde Keizer, M.C.A.; Flapper, S.D.P., Teunter, R.H. (2017). Condition-based maintenance policies for systems with multiple dependent components: A review. European Journal of Operational Research, http://dx.doi.org/10.1016/j.ejor.2017.02.044. 
[22] Panagiotidou, S. (2014). Joint optimization of spare parts ordering and maintenance policies for multiple identical items subject to silent failures. European Journal of Operational Research, 235, 300-314.

[23] Ross, S. (1996). Stochastic processes, 2nd edition. John Wiley \& Sons, New York.

[24] Satow, T.; Osaki, S. (2003). Optimal replacement policies for a two-unit system with shock damage interaction. Computers and Mathematics with Applications, 46, $1129-1138$.

[25] Scarf, P.A. and Deara, M. (1998). On the development and application of maintenance policies for a two-component system with failure dependence. IMA Journal of Mathematics Applied in Business \& Industry, 9, 91-107.

[26] Scarf, P.A. and Deara, M. (2003). Block replacement policies for a two-component system with failure dependence. Naval Research Logistics, 50, 70-87.

[27] Scarf, P.A.; Dwight, R. and Al-Musrati, A. (2005). On reliability criteria and the implied cost of failure for a maintained component. Reliability Engineering \& System Safety, 89, 199-207.

[28] Scarf, P.A. and Cavalcante, C.A.V. (2010). Hybrid block replacement and inspection policies for a multi-component system with heterogeneous component lives. European Journal of Operational Research, 206(2), 384-394.

[29] Sheu, S-H.; Sung, C-K; Hsu, T-S.; Chen Y-C. (2013). Age replacement policy for a two-unit system subject to non-homogeneous pure birth shocks. Applied Mathematical Modelling, 37, 7027-7036.

[30] Si, X.S.; Wang, W.; Hu, C.H. and Zhou, D.H. (2011). Remaining useful life estimation - A review on the statistical data driven approaches. European Journal of Operational Research, 213, 1-14.

[31] Sun, Y; Ma, L.; Mathew, J. (2009). Failure analysis of engineering systems with preventive maintenance and failure interactions. Computers \& Industrial Engineering, $57,539-549$.

[32] Taghipour, S., Banjevic, D., and Jardine, A. K. S. (2010). Periodic inspection optimization model for a complex repairable system. Reliability Engineering and System Safety, 95, 944-952. 
[33] Wang, H. (2002). A survey of maintenance policies of deteriorating systems. European Journal of Operational Research, 139, 469-489

[34] Wang, W. (1997). Subjective estimation of the delay time distribution in maintenance modelling. European Journal of Operational Research, 99, 516-529.

[35] Wang, W.; Banjevic, D., \& Pecht, M. (2010). A multi-component and multi-failure mode inspection model based on the delay time concept. Reliability Engineering and System Safety, 95, 912-920.

[36] Wang, W. (2012). An overview of the recent advances in delay-time-based maintenance modelling. Reliability Engineering and System Safety, 106, 165-178.

[37] Zequeira, R. and Bérenguer, C. (2005). On the inspection policy of a two-component parallel system with failure interaction. Réliability Engineering and System Safety, 88, 99-107. 